\title{
Effect of resting pressure on the estimate of cerebrospinal fluid outflow conductance
}

Kennet Andersson ${ }^{1 *}$, Nina Sundström³ ${ }^{3}$ Jan Malm², Anders Eklund ${ }^{1,3}$

\begin{abstract}
Background: A lumbar infusion test is commonly used as a predictive test for patients with normal pressure hydrocephalus and for evaluation of cerebrospinal fluid (CSF) shunt function. Different infusion protocols can be used to estimate the outflow conductance $\left(C_{\text {out }}\right)$ or its reciprocal the outflow resistance $\left(R_{\text {out }}\right)$, with or without using the baseline resting pressure, $P_{\mathrm{r}}$. Both from a basic physiological research and a clinical perspective, it is important to understand the limitations of the model on which infusion tests are based. By estimating $C_{\text {out }}$ using two different analyses, with or without $P_{r}$, the limitations could be explored. The aim of this study was to compare the $C_{\text {out }}$ estimates, and investigate what effect $P_{\mathrm{r}}$ had on the results.
\end{abstract}

Methods: Sixty-three patients that underwent a constant pressure infusion protocol as part of their preoperative evaluation for normal pressure hydrocephalus, were included (age $70.3 \pm 10.8$ years (mean \pm SD)). The analysis was performed without $\left(C_{\text {excl Pr }}\right)$ and with $\left(C_{\text {incl }} P_{r}\right) P_{r}$. The estimates were compared using Bland-Altman plots and paired sample $t$-tests $(p<0.05$ considered significant).

Results: Mean $C_{\text {out }}$ for the 63 patients was: $C_{\text {excl Pr }}=7.0 \pm 4.0$ (mean \pm SD) $\mu \mathrm{l} /(\mathrm{s} \mathrm{kPa})$ and $C_{\text {incl }} \operatorname{Pr}=9.1 \pm 4.3 \mu \mathrm{l} /(\mathrm{s}$ $\mathrm{kPa}$ ) and $R_{\text {out }}$ was $19.0 \pm 9.2$ and $17.7 \pm 11.3 \mathrm{mmHg} / \mathrm{ml} / \mathrm{min}$, respectively. There was a positive correlation between methods $(r=0.79, n=63, p<0.01)$. The difference, $\Delta C_{\text {out }}=-2.1 \pm 2.7 \mu l /(\mathrm{s} \mathrm{kPa})$ between methods was significant $(p<0.01)$ and $\Delta R_{\text {out }}$ was $1.2 \pm 8.8 \mathrm{mmHg} / \mathrm{ml} / \mathrm{min}$ ). The Bland-Altman plot visualized that the variation around the mean difference was similar all through the range of measured values and there was no correlation between $\Delta C_{\text {out }}$ and $C_{\text {out. }}$.

Conclusions: The difference between $C_{\text {out }}$ estimates, obtained from analyses with or without $P_{r}$, needs to be taken into consideration when comparing results from studies using different infusion test protocols. The study suggests variation in CSF formation rate, variation in venous pressure or a pressure dependent $C_{\text {out }}$ as possible causes for the deviation from the CSF absorption model seen in some patients.

\section{Background}

Patients with normal pressure hydrocephalus (NPH) are treated with and often improved by a cerebrospinal fluid (CSF) shunt that changes the dynamics of the CSF system [1-4]. In order to assist in the selection of patients likely to benefit from shunt surgery, predictive tests are performed [5]. One such test is the infusion test. It measures changes in intracranial pressure due to infusion or withdrawal of Ringer solution. For clinical interpretation, the relation between pressure and flow obtained during an infusion test must be quantified into

\footnotetext{
* Correspondence: kennet.andersson@radfys.umu.se

'Department of Radiation Sciences, Umeå University, Umeå, Sweden

Full list of author information is available at the end of the article
}

accessible parameters, i.e. a model of the CSF system is needed.

In the early seventies, Davson presented a model of the CSF absorption [6,7]. This has since been widely accepted and is used as one part of the model describing the dynamics of the CSF system:

$$
I_{\mathrm{a}}=\left(P_{\mathrm{ic}}-P_{\mathrm{d}}\right) C_{\text {out }}=\frac{P_{\text {ic }}-P_{\mathrm{d}}}{R_{\text {out }}}
$$

Thus, it states that the rate of absorption $\left(I_{\mathrm{a}}\right)$ is proportional to the difference between the pressure in the subarachnoid space $\left(P_{\text {ic }}\right)$ and venous pressure in dural sinus $\left(P_{\mathrm{d}}\right)$. The proportionality coefficient is the outflow conductance $\left(C_{\text {out }}\right)$, or its reciprocal, the outflow

\section{() Biomed Central}


resistance $\left(R_{\text {out }}\right) . C_{\text {out }}$ describes the ease of flow across the CSF outflow pathways. In addition to being used as a prognostic parameter for selecting patients responding to CSF shunt surgery, infusion measurement of $C_{\text {out }}$ is also used for evaluation of CSF shunt function [5,8-11].

To use equation (1) in the analysis of an infusion test, $P_{\mathrm{d}}$, which is difficult to measure, can be replaced by the measureable baseline resting pressure $P_{\mathrm{r}}$. To replace $P_{\mathrm{d}}$ with $P_{\mathrm{r}}$, three assumptions are needed, that $C_{\text {out }}$ is a physical property independent of pressure and that the variations in $P_{\mathrm{d}}$ and CSF formation rate, $I_{\mathrm{f}}$, during the infusion test are sufficiently small for $P_{\mathrm{d}}$ and $I_{\mathrm{f}}$ to be approximated as constants. If the variations in $P_{\mathrm{d}}, I_{\mathrm{f}}$ and $C_{\text {out }}$ are negligible, the relationship between steady state pressure and net infusion flow should be linear. Since a model is never better than the validity of its assumptions, it is important to understand the effects on estimated $C_{\text {out }}$ caused by unfulfilled assumptions.

There are different infusion protocols, one such is the constant pressure infusion $(\mathrm{CPI})$ protocol. It measures $P_{\mathrm{r}}$ and six elevated pressure levels together with corresponding net flow [12]. With this particular protocol, as opposed to the commonly used constant infusion protocol [13], a more detailed pressure/flow relationship can be plotted. As mentioned, data is expected to form a straight line throughout the pressure range with a trajectory through $P_{\mathrm{r}}$ and with the slope corresponding to $C_{\text {out }}$ (Figure 1 ). However, from clinical experience it is suspected that the regression line does not always pass through $P_{\mathrm{r}}$.

To understand the limitations of the current model used in infusion tests is important, both for basic physiological research and for clinical purposes. These limitations could be explored by comparing $C_{\text {out }}$ estimates calculated using two different analyses, one that included $P_{\mathrm{r}}$ and one that did not. The aim of this study was to investigate how the use of baseline resting pressure influences the estimate of $C_{\text {out }}$.

\section{Methods}

\section{Patient population}

The study population consisted of patients that underwent preoperative evaluation for NPH. All patients had an MRI that revealed ventriculomegaly (Evans ratio > 0.3 ) and they were without any visual obstruction to CSF flow. Sixty-three patients (age $70.3 \pm 10.8$ years (mean \pm SD), 18 women) underwent a CPI protocol. The study has been reviewed by the Regional Ethical Review Board in Umeå who concluded that there were no ethical problems with the project.

\section{Infusion apparatus and investigation}

The highly standardized infusion apparatus has been thoroughly described previously [12]. Two needles were inserted in the spinal canal while the patient was in the
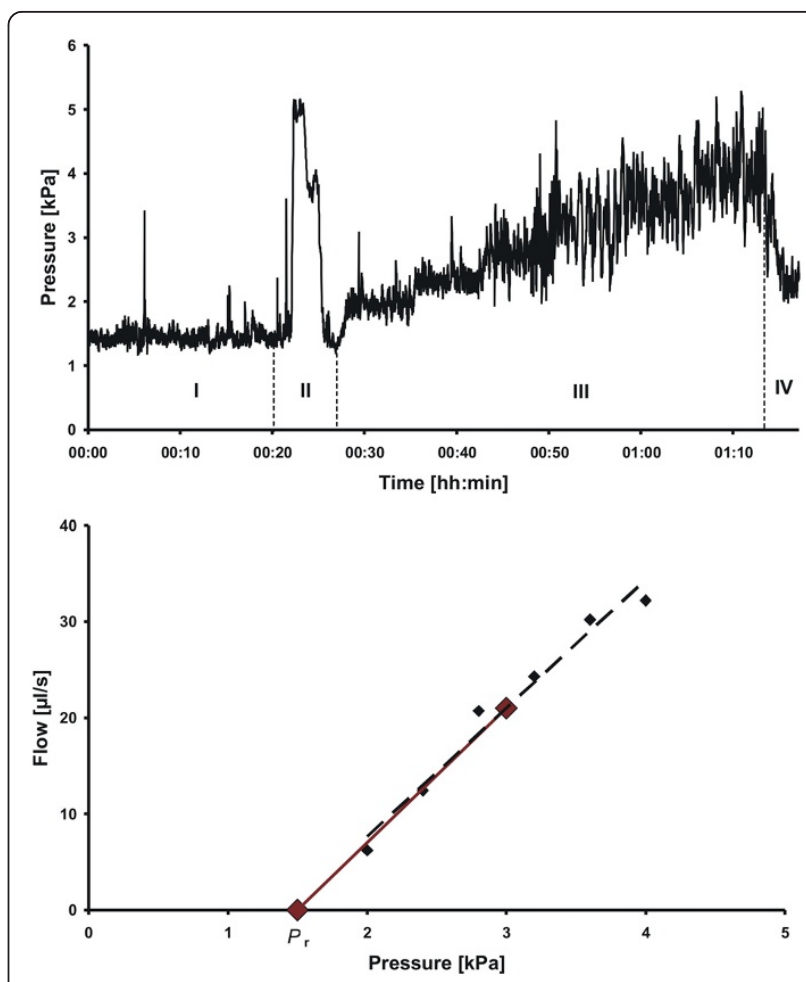

Figure 1 Upper plot of pressure against time for one experiment: the infusion investigation starts with measurement of $P_{\mathrm{r}}(\mathrm{I}), \mathrm{CSF}$ sampling with patient in sitting position (II), CPI protocol with six elevated pressure levels back in supine position (III) and a relaxation phase (IV). Lower plot of estimated flow against pressure: Results from the patient measurement illustrating the two analysis methods graphically. Lower red dot is measured $P_{r}$, upper red dot is mean of the six black dots which are measured flow and pressure from the elevated pressure levels. The dotted black regression line of the six elevated levels illustrate method 1 , the red line, connecting $P_{\mathrm{r}}$ and the mean of the elevated levels, illustrate method 2. The slopes of the lines give the $C_{\text {out }}$ estimates respectively.

sitting position, one needle was used for pressure measurement and the other for infusion or withdrawal of Ringer solution. The patient was placed in the supine position and the zero-pressure reference level was placed at the level of the auditory meatus. The investigation is illustrated in Figure 1. First, $P_{\text {ic }}$ was measured during 15-20 minutes of rest, and $P_{\mathrm{r}}$ was calculated as the mean $P_{\text {ic }}$ over the last five minutes. To ensure a stable measurement of $P_{\mathrm{r}}$, the patient was lying comfortably in supine position during the investigation, the importance of minimizing leakage during lumbar puncture was accentuated to the physician and the routine sample of CSF was taken after the measurement of $P_{\mathrm{r}}$. Following the $P_{\mathrm{r}}$ measurement, the CPI protocol was initiated. $P_{\text {ic }}$ was increased to six, consecutive, predetermined pressure levels lasting seven minutes each (Figure 1) followed by a spontaneous relaxation phase. 


\section{Estimation of $\mathrm{C}_{\text {out }}$}

The CSF absorption is estimated from Davson's equation (1). The two estimation methods used in this study are described below and illustrated in Figure 1 and Figure 2. They are derived from the model of CSF absorption and a CSF system in steady state. The assumption of conservation of fluid in the CSF system can be stated as

$$
I_{\mathrm{f}}+I_{\mathrm{ext}}=I_{\mathrm{a}}+I_{\mathrm{s}}
$$

where $I_{\mathrm{f}}$ is the formation rate, $I_{\text {ext }}$ is the infusion rate of a possible external infusion, $I_{\mathrm{a}}$ is the rate of absorption and $I_{\mathrm{s}}$ is the rate of change of fluid stored in the system. The normal unperturbed baseline resting pressure, $P_{\mathrm{r}},\left(I_{\mathrm{s}}\right.$ and $I_{\text {ext }}$ equal to zero $)$ of the patient is defined as

$$
P_{\mathrm{r}}=P_{\mathrm{d}}+\frac{I_{\mathrm{f}}}{C_{\text {out }}}
$$

When in steady state during an infusion test, $I_{\mathrm{a}}=I_{\text {ext }}$ $+I_{\mathrm{f}}$, see equation (2). Combining this with equations (1) and (3), the relation between $I_{\text {ext }}$ and $P_{\text {ic }}$ is

$$
I_{\text {ext }}=C_{\text {out }}\left(P_{\text {ic }}-P_{\mathrm{r}}\right)
$$

\section{Method 1, analysis without $\mathrm{P}_{\mathrm{r}}$}

On each of the six elevated pressure levels, mean $P_{\text {ic }}$ as well as the net inflow $\left(I_{\text {ext }}\right)$ needed to maintain a constant $P_{\text {ic }}$ was measured. The relation between $I_{\text {ext }}$ and $P_{\text {ic }}$ was

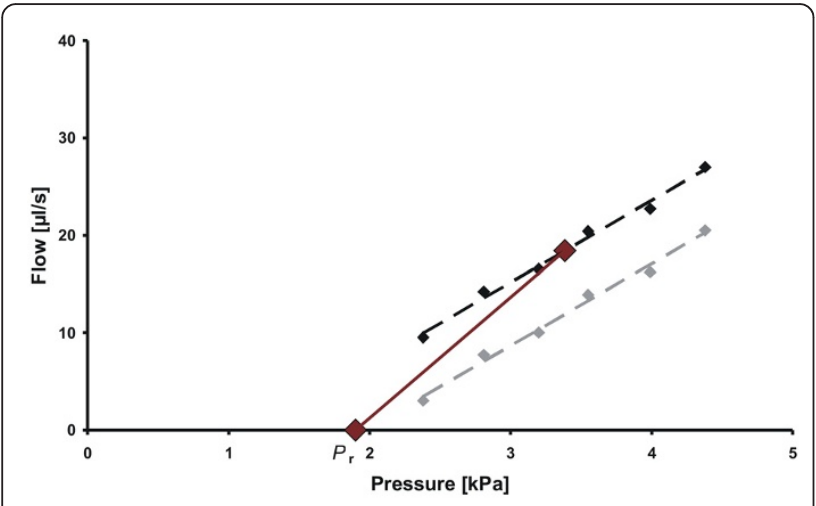

Figure 2 Results illustrating a patient with large difference between methods. Lower red dot is measured $P_{r}$ and upper large red dot is mean of the six black dots. The dotted black line is the estimate of $C_{\text {excl Pr, }}$ the red line is $C_{\text {incl }}$ Pr. Lower grey points with regression line illustrate a possible result without needed extra net flow. This typical pattern of extra net flow was visually observed for approximately one third of the patients.

$$
I_{\text {ext }}=C_{\text {exclPr }} P_{\text {ic }}+\text { constant }
$$

$C_{\text {excl Pr }}$ was estimated as the slope of the linear regression between $I_{\text {ext }}$ and $P_{\text {ic }}$ using the six elevated pressure levels [12,14] (Figure 1).

\section{Method 2, analysis with $\mathrm{Pr}_{\mathrm{r}}$}

Pressure and flow from all six elevated levels, but without using the $P_{\mathrm{r}}$, were averaged into one pressure and flow point $\left(\bar{P}_{\text {ic }}\right.$ and $I_{\text {ext }}$ respectively). $C_{\text {incl Pr was calculated as }}$

$$
C_{\text {ind Pr }}=\frac{\bar{I}_{\text {ext }}}{\bar{P}_{\text {ic }}-P_{\mathrm{r}}}
$$

i.e. a line was drawn between $P_{\mathrm{r}}$ and $\bar{P}_{\mathrm{ic}}$ and the slope corresponded to $C_{\text {incl } \operatorname{Pr}}$ (Figure 1). The classic Katzman method of estimating $C_{\text {out }}$ during a constant infusion is achieved by dividing the mean flow with the difference between resting pressure and a pressure plateau [13]. The method for $C_{\text {incl }}$ Pr simulates that approach and uses the same formula.

\section{Statistics}

Pearson's correlation coefficient was used for correlation analysis. The two estimates of $C_{\text {out }}$ were compared using Bland-Altman plots and paired sample $t$-tests, $p<0.05$ was considered significant.

\section{Results}

A typical infusion investigation is shown in Figure 1 with corresponding $C_{\text {out }}$ from the two methods. The mean outflow conductance for the 63 patients was $C_{\text {excl }}$ $\mathrm{Pr}=7.0 \pm 4.0($ mean $\pm \mathrm{SD}) \mu \mathrm{l} /(\mathrm{s} \mathrm{kPa})\left(R_{\text {excl } \operatorname{Pr}}=19.0 \pm\right.$ $9.2 \mathrm{mmHg} / \mathrm{ml} / \mathrm{min})$ and $C_{\text {incl } \operatorname{Pr}}=9.1 \pm 4.3 \mu \mathrm{l} /(\mathrm{s} \mathrm{kPa})$ $\left(R_{\text {incl Pr }}=17.7 \pm 11.3 \mathrm{mmHg} / \mathrm{ml} / \mathrm{min}\right)$ respectively. There was a positive correlation between the two methods $(\mathrm{r}=0.79, \mathrm{n}=63, p<0.01)$. The paired difference between estimation methods $\left(\Delta C_{\text {out }}=C_{\text {excl Pr }}-C_{\text {incl Pr }}\right)$ was significant, $\Delta C_{\text {out }}=-2.1 \pm 2.7 \mu \mathrm{l} /(\mathrm{s} \mathrm{kPa}), \mathrm{n}=63$, $p<0.01\left(\Delta R_{\text {out }}=1.2 \pm 8.8 \mathrm{mmHg} / \mathrm{ml} / \mathrm{min}\right)$. The SD of $\Delta C_{\text {out }}$ was $13 \%$ of the measurement range. Figure 2 illustrates a case where the difference between methods was large, $\Delta C_{\text {out }}=4.1 \mu \mathrm{l} /(\mathrm{s} \mathrm{kPa})$, is shown. Two phases were identified: 1 . a net flow needed to raise the pressure from $P_{\mathrm{r}}$ to the first level, 2. a pattern following a straight line from the first level to the sixth level.

The Bland-Altman plot in Figure 3 shows $\Delta C_{\text {out }}$ plotted against the mean of the two analysis methods. The variation around the mean difference in $C_{\text {out }}$ was similar all through the range of measured pressures and there was no correlation between $\Delta C_{\text {out }}$ and $C_{\text {out }}$. A corresponding plot for $R_{\text {out }}$ is given in Figure 4 . 


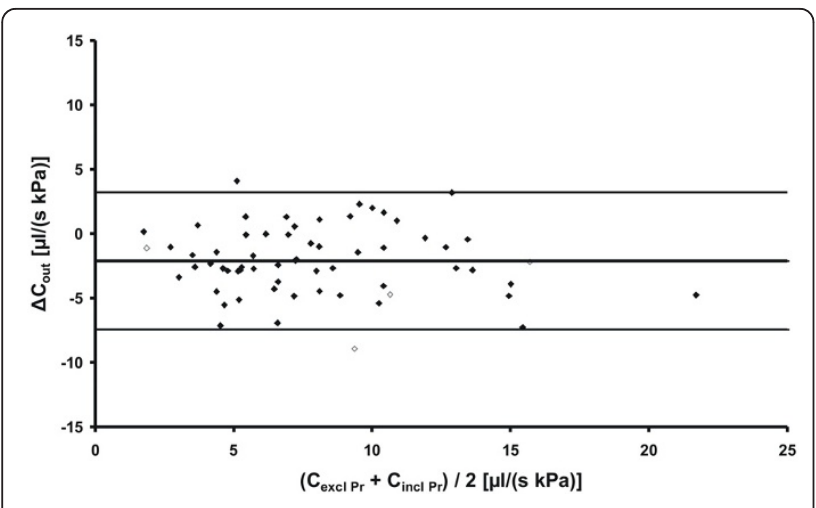

Figure $3 \mathrm{~A}$ Bland-Altman plot of the two analysis methods for $C_{\text {out }}$ showing the difference $\Delta C_{\text {out, }}$ vs. the average of the two methods for all subjects. The lines are calculated as mean \pm 1.96 SD. The open diamonds represent subjects with marked B-waves during $P_{\mathrm{r}}$ measurement.

\section{Discussion}

This study investigated two analysis methods for estimating $C_{\text {out }}$, with or without $P_{\mathrm{r}}$. The significant difference between the two methods (Figure 3) should be considered when comparing $C_{\text {out }}$ in studies using different methods and when setting threshold values for shunting. The correlation between methods was in the same range as between $C_{\text {excl Pr }}$ and $C_{\text {out }}$ from a previous study [15]. It should be noted that the difference between the two methods was small and similar to what has been found for repeated infusion protocols $[12,15,16]$, therefore one has to be careful with regard to any clinical implications. Most analysis methods for infusion tests are based on the model and basic assumptions described in this paper, and current development of new analysis methods for pressure-controlled infusion will, as opposed to the CPI method used today, rely on

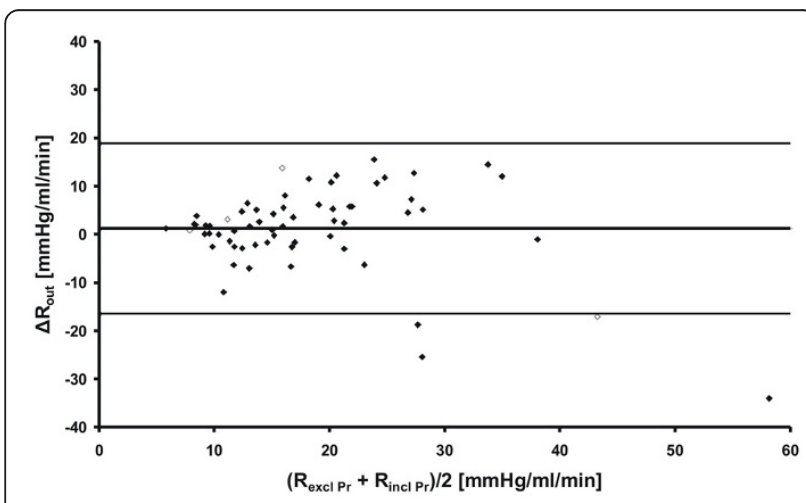

Figure $4 \mathrm{~A}$ Bland-Altman plot of the two analysis methods for $R_{\text {out }}$ showing the difference $\Delta R_{\text {out }}$ vs. the average of the two methods. The lines are calculated mean $\pm 1.96 \mathrm{SD}$. The open diamonds represent subjects with marked B-waves during $P_{r}$ measurement.
$P_{\text {r }}$ [17]. It is therefore important to investigate the limitations of these assumptions and the effects they have on calculated $C_{\text {out }}$.

The difference that was found depending on whether or not $P_{\mathrm{r}}$ was used in the estimation of $C_{\text {out }}$, (Figure 3 ), could be explained by several underlying causes. The infusion test analysis based on equation (1) assumes that $P_{\mathrm{d}}$ and $I_{\mathrm{f}}[18]$ are constant, but if they varied during the investigation, both $P_{\mathrm{r}}$ and the estimation of $C_{\text {out }}$ would be affected. A potential explanation could be that the infusion of Ringer solution caused a physiological response with a reduction in $P_{\mathrm{d}}$ and/or $I_{\mathrm{f}}$ which would result in an increase of needed inflow as observed in this study (Figure 2), giving rise to the systematic difference in estimated $C_{\text {out }}$ depending on whether or not $P_{\mathrm{r}}$ was used. Another assumption was that $C_{\text {out }}$ is constant and pressure independent. This assumption has been based on visual inspection or correlation coefficients of the pressure/flow relationship [19-24]. Specifically, a linear relationship was shown for a pressure interval of 0.7-1.6 $\mathrm{kPa}$ above $P_{\mathrm{r}}$ [25], but that study focused on the use of $C_{\text {excl Pr }}$ and did not analyse the relationship down to $P_{\mathrm{r}}$. Other studies have proposed a nonlinear relationship between pressure and flow [26-28]. These studies suggested a continuously pressure dependent $C_{\text {out }}$ while in the present study, the results suggest that for certain patients (Figure 2), there was a higher $C_{\text {out }}$ in the vicinity of $P_{\mathrm{r}}$ followed by a pressure independent $C_{\text {out }}$. This could be explained by an active CSF outflow transport that starts when the system is perturbed by infusion, but with an absorption rate that is independent of further increases in pressure. This would indicate that the CSF outflow in the vicinity of $P_{\mathrm{r}}$ in some cases may differ from the Davson equation.

It was not possible to deduce from this study which of $I_{\mathrm{f}}, P_{\mathrm{d}}$ and a pressure independent $C_{\text {out }}$ was the major contributor to the systematic difference in results. The authors believe that the Davson equation is valid and that the deviation came from variations in $P_{\mathrm{d}}$ and/or $I_{\mathrm{f}}$ during the infusion. Monitoring of variation in central venous pressure during infusion tests could be a possible way forward. In addition to the systematic difference between methods, there was also a variation around the mean. This variation was probably mainly caused by the vascular effects on the CSF system (Figure 3). Vasomotion can cause large volume variations on the arterial side which in turn induce large pressure variations, e.g. B-waves [29]. The relatively small flows involved during an infusion test in comparison with these effects, will make the estimation of $C_{\text {out }}$ challenging. The steadystate analysis approach assumes that the dynamics of the system will be sufficiently suppressed by averaging over the 7 minutes of measurement time. However, the system dynamics for many patients can include 
components with potential to violate this assumption, e. g. B-waves or plateau waves, that can cause a reduction in accuracy of the estimated pressures and flows for the elevated levels $[29,30]$. These comparatively large physiological variations will also influence measured $P_{\mathrm{r}}$. Visual inspection of the $P_{\mathrm{r}}$ measurements showed that four patients had marked B-waves. One of which was the subject with the highest difference between methods while the other three were method independent (Figure 3). Furthermore, pressure that had not stabilised enough during its 15-20 min baseline measurement, would also affect $P_{\mathrm{r}}$. This could be caused by apprehension of the patient. Another possibility was a slow formation rate unable to compensate for the loss of CSF during lumbar puncture. To avoid this, a routine was followed in order to obtain as reliable estimates as possible (see Methods section). Results of repeated measurements in the same patient with consecutive CPI and constant infusion protocols suggest that the vascular effects limit the expected precision for measurements with current infusion tests to approximately $2 \mu \mathrm{l} /(\mathrm{s} \mathrm{kPa})(\mathrm{SD})[12,15,16]$. We interpret this as an inherent characteristic of the vascular and CSF system that limits the expected repeatability independently of which infusion method that is used.

Since $C_{\text {incl }}$ Pr uses an average value it will be less sensitive to physiological variations at the lowest or highest pressure levels. On the other hand it is dependent on $P_{\mathrm{r}}$, and an error in this parameter will have a major impact on the estimated $C_{\text {out }}$, equation (6). Thus, the accuracy of estimated $P_{\mathrm{r}}$ becomes essential. Furthermore, if results are compared with results from the constant infusion protocol with either static analysis according to Katzman [13] or dynamic analysis [31], $C_{\text {incl Pr }}$ should be used. Until future clinical studies have investigated the pressure/flow relationship in the vicinity of $P_{\mathrm{r}}$ in more detail and its pathophysiological importance have been established, both methods are still relevant. An erroneous flow measurement could produce the shift upwards in flow (Figure 2). However, careful calibration and testing of the equipment on experimental set-up was performed [12,17], and these types of errors have not been observed.

\section{Conclusions}

Using $P_{\mathrm{r}}$ for estimating $C_{\text {out }}$ produced a higher estimated $C_{\text {out }}$. Possible causes for a deviation from the model of CSF absorption in some patients were a variation in formation rate or venous pressure or a pressure dependent $C_{\text {out }}$. The observed difference needs to be taken into consideration when setting threshold values for shunting and when comparing results from studies using different infusion test protocols.

\section{List of abbreviations}

NPH: Normal pressure hydrocephalus; CSF: Cerebrospinal fluid; $P_{\mathrm{ic}}$ Intracranial pressure; $P_{\mathrm{r}}$ : Resting pressure; $P_{\mathrm{d}}$ : Venous pressure in dural sinus; $l_{a}$ : Absorption rate of CSF; lf: Formation rate of CSF; I lext: Rate of external infusion; Is: CSF stored in system; $\mathrm{P}^{-} \mathrm{ic}$ : Average pressure; I'ic; Average flow; $C_{\text {excl pr: }}$ Outflow conductance estimated by method 1 , without $P_{r} ; C_{\text {incl pr: }}$ : Outflow conductance estimated by method 2, with $P_{r} ; \Delta C_{\text {out }}$ : Difference in conductance between methods

\section{Acknowledgements}

The study was funded by the Objective 2 Norra Norrland-EU Structural Fund, the Swedish research council, Vinnova, and the Foundation for Strategic Research through their joint initiative Biomedical Engineering for Better Health.

\section{Author details}

${ }^{1}$ Department of Radiation Sciences, Umeå University, Umeå, Sweden. ${ }^{2}$ Department of Clinical Neuroscience, Umeå University, Umeå, Sweden. ${ }^{3}$ Centre of Biomedical Engineering and Physics, Umeå University, Umeå, Sweden.

\section{Authors' contributions}

All authors participated in the conception and design of the study, collection of data, statistical analysis and critically revised the article, reviewed the final version of the manuscript and approved it for submission.

\section{Competing interests}

Drs. Sundström, Malm, and Eklund have a patent interest in the in-housedeveloped infusion apparatus used in the study. Likvor AB has acquired the patent rights for commercialization.

Received: 23 December 2010 Accepted: 7 March 2011

Published: 7 March 2011

\section{References}

1. Malm J, Kristensen B, Karlsson T, Fagerlund M, Elfverson J, Ekstedt J: The predictive value of cerebrospinal fluid dynamic tests in patients with idiopathic adult hydrocephalus syndrome. Arch Neurol 1995, 52:783-789.

2. Tullberg M, Jensen C, Ekholm S, Wikkelso C: Normal pressure hydrocephalus: vascular white matter changes on MR images must not exclude patients from shunt surgery. AJNR Am J Neuroradiol 2001, 22:1665-1673.

3. Boon AJ, Tans JT, Delwel EJ, Egeler-Peerdeman SM, Hanlo PW, Wurzer HA, Avezaat CJ, de Jong DA, Gooskens RH, Hermans J: Dutch normal-pressure hydrocephalus study: prediction of outcome after shunting by resistance to outflow of cerebrospinal fluid. J Neurosurg 1997, 87:687-693.

4. Tisell M, Tullberg M, Hellstrom P, Edsbagge M, Hogfeldt M, Wikkelso C: Shunt surgery in patients with hydrocephalus and white matter changes. J Neurosurg 2011.

5. Marmarou A, Bergsneider M, Klinge P, Relkin N, Black PM: The Value of Supplemental Prognostic Tests for the Preoperative Assessment of Idiopathic Normal-pressure Hydrocephalus. Neurosurgery 2005, 57:17-28.

6. Davson H, Domer FR, Hollingsworth JR: The mechanism of drainage of the cerebrospinal fluid. Brain 1973, 96:329-336.

7. Davson $H$, Hollingsworth $G$, Segal MB: The mechanism of drainage of the cerebrospinal fluid. Brain 1970, 93:665-678.

8. Czosnyka M, Whitehouse H, Smielewski P, Simac S, Pickard JD: Testing of cerebrospinal compensatory reserve in shunted and non-shunted patients: a guide to interpretation based on an observational study. $J$ Neurol Neurosurg Psychiatry 1996, 60:549-558.

9. Eklund A, Lundkvist B, Koskinen LO, Malm J: Infusion technique can be used to distinguish between dysfunction of a hydrocephalus shunt system and a progressive dementia. Med Biol Eng Comput 2004, 42:644-649.

10. Eklund A, Smielewski P, Chambers I, Alperin N, Malm J, Czosnyka M, Marmarou A: Assessment of cerebrospinal fluid outflow resistance. Med Biol Eng Comput 2007, 45:719-735.

11. Lundkvist B, Koskinen LO, Birgander R, Eklund A, Malm J: Cerebrospinal fluid dynamics and long-term survival of the Strata((R)) valve in idiopathic normal pressure hydrocephalus. Acta Neurol Scand 2010. 
12. Andersson N, Malm J, Backlund T, Eklund A: Assessment of cerebrospinal fluid outflow conductance using constant-pressure infusion-a method with real time estimation of reliability. Physiol Meas 2005, 26:1137-1148.

13. Katzman R, Hussey F: A simple constant-infusion manometric test for measurement of CSF absorption. I. Rationale and method. Neurology 1970, 20:534-544.

14. Andersson K, Manchester IR, Andersson N, Shiriaev AS, Malm J, Eklund A: Assessment of cerebrospinal fluid outflow conductance using an adaptive observer-experimental and clinical evaluation. Physiol Meas 2007, 28:1355-1368.

15. Sundstrom N, Andersson K, Marmarou A, Malm J, Eklund A: Comparison between 3 infusion methods to measure cerebrospinal fluid outflow conductance. J Neurosurg 113:1294-1303.

16. Borgesen SE, Albeck MJ, Gjerris F, Czosnyka M, Laniewski P: Computerized infusion test compared to steady pressure constant infusion test in measurement of resistance to CSF outflow. Acta Neurochir (Wien) 1992, 119:12-16.

17. Andersson K, Manchester IR, Malm J, Eklund A: Real-time estimation of cerebrospinal fluid system parameters via oscillating pressure infusion. Med Biol Eng Comput 48:1123-1131.

18. Cutler RW, Page L, Galicich J, Watters GV: Formation and absorption of cerebrospinal fluid in man. Brain 1968, 91:707-720.

19. Ekstedt J: CSF hydrodynamic studies in man. 1. Method of constant pressure CSF infusion. J Neurol Neurosurg Psychiatry 1977, 40:105-119.

20. Ekstedt J: CSF hydrodynamic studies in man. 2. Normal hydrodynamic variables related to CSF pressure and flow. I Neurol Neurosurg Psychiatry 1978, 41:345-353.

21. Portnoy HD, Croissant PD: A practical method for measuring hydrodynamics of cerebrospinal fluid. Surg Neurol 1976, 5:273-277.

22. Borgesen $\mathrm{SE}$, Gjerris $\mathrm{F}$ : The predictive value of conductance to outflow of CSF in normal pressure hydrocephalus. Brain 1982, 105:65-86.

23. Borgesen SE, Gjerris F, Srensen SC: The resistance to cerebrospinal fluid absorption in humans. A method of evaluation by lumbo-ventricular perfusion, with particular reference to normal pressure hydrocephalus. Acta Neurol Scand 1978, 57:88-96.

24. Sklar FH, Beyer CW, Ramanathan M, Elashvili I, Cooper PR, Clark WK: Servocontrolled lumbar infusions: a clinical tool for the determination of CSF dynamics as a function of pressure. Neurosurgery 1978, 3:170-175.

25. Andersson N, Malm J, Eklund A: Dependency of cerebrospinal fluid outflow resistance on intracranial pressure. J Neurosurg 2008, 109:918-922.

26. Meier U, Kiefer M, Bartels P: The ICP-dependency of resistance to cerebrospinal fluid outflow: a new mathematical method for CSFparameter calculation in a model with H-TX rats. J Clin Neurosci 2002, 9:58-63.

27. Meier $U$, Bartels $P$ : The importance of the intrathecal infusion test in the diagnosis of normal pressure hydrocephalus. J Clin Neurosci 2002, 9:260-267.

28. Tychmanowicz K, Czernicki Z, Pawlowski G, Stepinska G: ICP dependent changes of CSF outflow resistance. Acta Neurochir (Wien) 1992, 117:44-47.

29. Lundberg N: Continuous recording and control of ventricular fluid pressure in neurosurgical practice. Acta Psychiatr Scand 1960, 36(Suppl 149):1-193.

30. Lenfeldt N, Andersson N, Agren-Wilsson A, Bergenheim AT, Koskinen LO, Eklund A, Malm J: Cerebrospinal fluid pulse pressure method: a possible substitute for the examination of B waves. J Neurosurg 2004, 101:944-950.

31. Czosnyka M, Batorski L, Laniewski P, Maksymowicz W, Koszewski W, Zaworski W: A computer system for the identification of the cerebrospinal compensatory model. Acta Neurochir (Wien) 1990, 105:112-116.

doi:10.1186/2045-8118-8-15

Cite this article as: Andersson et al: Effect of resting pressure on the estimate of cerebrospinal fluid outflow conductance. Fluids and Barriers of the CNS 2011 8:15.

\section{Submit your next manuscript to BioMed Central and take full advantage of:}

- Convenient online submission

- Thorough peer review

- No space constraints or color figure charges

- Immediate publication on acceptance

- Inclusion in PubMed, CAS, Scopus and Google Scholar

- Research which is freely available for redistribution 